\title{
On the Mazur-Ulam problem in non-Archimedean fuzzy 2-normed spaces
}

Heejeong Koh and Dongseung Kang*

${ }^{*}$ Correspondence:

dskang@dankook.ac.kr

Department of Mathematics

Education, Dankook University, 126,

Jukjeon, Suji, Yongin, Gyeonggi

448-701, South Korea

\begin{abstract}
We study the notion of non-Archimedean fuzzy 2-normed space over a non-Archimedean field and prove that the Mazur-Ulam theorem holds under some conditions in the non-Archimedean fuzzy 2-normed space.

MSC: Primary 46S10; secondary 47S10; 26E30; $12 \mathrm{~J} 25$

Keywords: Mazur-Ulam theorem; non-Archimedean field; non-Archimedean fuzzy normed spaces; isometry; fuzzy 2-isometry
\end{abstract}

\section{Introduction}

A mapping $f: X \longrightarrow Y$ is called an isometry if $f$ satisfies

$$
d_{Y}(f(x), f(y))=d_{X}(x, y)
$$

for all $x, y \in X$, where $d_{X}(\cdot, \cdot)$ and $d_{Y}(\cdot, \cdot)$ denote the metrics in the spaces $X$ and $Y$, respectively.

The theory of isometric mappings originated in the classical paper [1] by Mazur and Ulam in 1932.

Mazur-Ulam theorem Every isometry $f$ of a normed real linear space $X$ onto a normed real linear space is a linear mapping up to translation, that is, $x \mapsto f(x)-f(0)$ is linear, which amounts to the definition that $f$ is affine.

The Mazur-Ulam theorem is not true for a normed complex vector space. In addition, the onto assumption is also essential. Without this assumption, Baker [2] proved that an isometry from a normed real linear space into a strictly convex normed real linear space is affine.

Gähler [3, 4] introduced a new approach for a theory of 2-norm and $n$-norm on a linear space. Chu [5] studied the Mazur-Ulam theorem in linear 2-normed spaces. Recently, Moslehian and Sadeghi [6] introduced the Mazur-Ulam theorem in the non-Archimedean strictly convex normed spaces. Moreover, Mirmostafaee and Moslehian [7] introduced a non-Archimedean fuzzy norm on a linear space over a non-Archimedean field. In particular, Amyari and Sadeghi [8] proved Mazur-Ulam theorem under the condition of strict convexity in non-Archimedean 2-normed spaces.

In 1984, Katsaras [9] and Wu and Fang [10] introduced the notion of fuzzy norm, and also Wu and Fang gave the generalization of the Kolmogoroff normalized theorem for a

○2013 Koh and Kang; licensee Springer. This is an Open Access article distributed under the terms of the Creative Commons Attribution License (http://creativecommons.org/licenses/by/2.0), which permits unrestricted use, distribution, and reproduction in any medium, provided the original work is properly cited. 
fuzzy topological linear space. In addition, fuzzy $n$-normed linear spaces were studied by Narayanan and Vijayabalaji; see [11].

In this paper, we investigate the notion of non-Archimedean fuzzy 2-normed space over a linear ordered non-Archimedean field and prove that Mazur-Ulam theorem holds under some conditions in the non-Archimedean fuzzy 2-normed space.

Definition 1.1 A non-Archimedean field is a field $\mathcal{K}$ equipped with a (valuation) function from $\mathcal{K}$ into $[0, \infty)$ satisfying the following properties:

(1) $|a| \geq 0$ and equality holds if and only if $a=0$,

(2) $|a b|=|a||b|$,

(3) $|a+b| \leq \max \{|a|,|b|\}$

for all $a, b \in \mathcal{K}$.

Clearly, $|1|=|-1|=1$ and $|n| \leq 1$ for all $n \in \mathbb{N}$. An example of a non-Archimedean valuation is the function $|\cdot|$ taking everything except 0 into 1 and $|0|=0$; see [12]. We call it a non-Archimedean trivial valuation. Also, the most important examples of nonArchimedean spaces are $p$-adic numbers; see [7].

Definition 1.2 Let $X$ be a linear space over a field $\mathcal{K}$ with a non-Archimedean valuation $|\cdot|$. A function $\|\cdot\|: X \times X \longrightarrow[0, \infty)$ is said to be a non-Archimedean 2-norm if it satisfies the following properties:

(1) $\|x, y\|=0$ if and only if $x, y$ are linearly dependent,

(2) $\|x, y\|=\|y, x\|$,

(3) $\|c x, y\|=|c|\|x, y\|$,

(4) $\|x, y+z\| \leq \max \{\|x, y\|,\|x, z\|\}$

for all $x, y, z \in X$ and $c \in \mathcal{K}$. Then $(X,\|\cdot\|)$ is called a non-Archimedean 2-normed space.

Definition 1.3 Let $X$ be a linear space over a field $\mathcal{K}$ with a non-Archimedean valuation $|\cdot|$. A function $N: X^{2} \times \mathbb{R} \longrightarrow[0,1]$ is said to be a non-Archimedean fuzzy 2-norm on $X$ if for all $x, y \in X$ and all $s, t \in \mathbb{R}$,

(N1) $N(x, y, t)=0$ for $t \leq 0$,

(N2) for $t>0, N(x, y, t)=1$ if and only if $x$ and $y$ are linearly dependent,

(N3) $N(x, y, t)=N(y, x, t)$,

(N4) $N(x, c y, t)=N\left(y, x, \frac{t}{|c|}\right)$ for $c \neq 0$,

(N5) $N(x, y+z, \max \{s, t\}) \geq \min \{N(x, y, s), N(x, z, t)\}$,

(N6) $N(x, y, *)$ is a nondecreasing function of $\mathbb{R}$ and $\lim _{t \rightarrow \infty} N(x, y, t)=1$.

The pair $(X, N)$ is called a non-Archimedean fuzzy 2-normed space.

The property (N4) implies that $N(-x, y, t)=N(x, y, t)$ for all $x, y \in X$ and $t>0$. It is easy to show that (N5) is equivalent to the following condition:

$$
N(x, y+z, t) \geq \min \{N(x, y, t), N(x, z, t)\} \quad \text { for all } x, y, z \in X \text { and } t \in \mathbb{R} \text {. }
$$

Example 1.4 Let $(X,\|\cdot, \cdot\|)$ be a non-Archimedean 2-normed space. Define

$$
N(x, y, t)= \begin{cases}\frac{t}{t+\|x, y\|} & \text { when } t>0, t \in \mathbb{R}, \\ 0 & \text { when } t \leq 0,\end{cases}
$$

where $x, y \in X$. Then $(X, N)$ is a non-Archimedean fuzzy 2-normed space. 
Definition 1.5 A non-Archimedean fuzzy 2-normed space is said to be strictly convex if $N(x, y+z, \max \{s, t\})=\min \{N(x, y, s), N(x, z, t)\}$ and $N(x, y, s)=N(x, z, t)$ imply $y=z$ and $s=t$.

Definition 1.6 Let $(X, N)$ and $(Y, N)$ be two non-Archimedean fuzzy 2-normed spaces. We call $f:(X, N) \longrightarrow(Y, N)$ a fuzzy 2-isometry if $N(a-c, b-c, t)=N(f(a)-f(c), f(b)-$ $f(c), t)$ for all $a, b, c \in X$ and $t>0$.

Definition 1.7 Let $X$ be a non-Archimedean fuzzy 2-normed space, and let $a, b, c$ be mutually disjoint elements of $X$. Then $a, b$ and $c$ are said to be collinear if $b-c=r(a-c)$ for some real number $r$.

We denote the set of all elements of $\mathcal{K}$ whose norms are 1 by $\mathcal{C}$, that is,

$$
\mathcal{C}=\{r \in \mathcal{K}|| r \mid=1\} .
$$

\section{Main results}

Lemma 2.1 Let $(X, N)$ be a non-Archimedean fuzzy 2-normed space over a linear ordered non-Archimedean field $\mathcal{K}$. Then

$$
N(x, y, t)=N(x, y+r x, t) \quad \text { for all } r \in \mathcal{K} \text {. }
$$

Proof Let $x, y \in X$ and let $r \in \mathcal{K}$. Without loss of generality, we may assume $t>0$. Then

$$
N(x, y+r x, t) \geq \min \{N(x, y, t), N(x, r x, t)\}=N(x, y, t) .
$$

Conversely,

$$
\begin{aligned}
N(x, y, t) & =N(x, y+r x-r x, t) \geq \min \{N(x, y+r x, t), N(x, r x, t)\} \\
& =N(x, y+r x, t) .
\end{aligned}
$$

Thus $N(x, y, t)=N(x, y+r x, t)$ for all $r \in \mathcal{K}$.

Lemma 2.2 Let $(X, N)$ be a non-Archimedean fuzzy 2-normed space over a linear ordered non-Archimedean field $\mathcal{K}$ with $\mathcal{C}=\left\{2^{n} \mid n \in \mathbb{Z}\right\}$, and let $a, b, c \in X$ and $t>0$. Suppose that $X$ is strictly convex. Then $\alpha=\frac{a+b}{2}$ is the unique element of $X$ such that

$$
N(a-c, a-\alpha, t)=N(b-\alpha, b-c, t)=N(a-c, b-c, t),
$$

where $a, b$ and $\alpha$ are collinear.

Proof Let $\alpha=\frac{a+b}{2} \in X$ and $t>0$. By Lemma 2.1, we have

$$
\begin{aligned}
N(a-c, a-\alpha, t) & =N\left(a-c, a-\frac{a+b}{2}, t\right) \\
& =N\left(a-c, \frac{a-b}{2}, t\right)
\end{aligned}
$$




$$
\begin{aligned}
& =N(a-c, a-b,|2| t) \\
& =N(a-c, a-b, t) \\
& =N(a-c, b-c, t) .
\end{aligned}
$$

Similarly,

$$
\begin{aligned}
N(b-\alpha, b-c, t) & =N\left(b-\frac{a+b}{2}, b-c, t\right)=N(b-a, b-c, t) \\
& =N(a-c, b-c, t) .
\end{aligned}
$$

Hence we have $N(a-c, a-\alpha, t)=N(a-c, b-c, t)=N(b-\alpha, b-c, t)$, that is, the existence part holds. To show the uniqueness part, assume that $\beta$ is an element of $X$ such that

$$
N(a-c, a-\beta, t)=N(b-\beta, b-c, t)=N(a-c, b-c, t),
$$

where $a, b$ and $\beta$ are collinear. Since $a, b$ and $\beta$ are collinear, there exists a real number $s$ such that

$$
\beta=s a+(1-s) b
$$

We may assume $s \neq 0$ and $s \neq 1$.

$$
\begin{aligned}
N(a-c, b-c, t) & =N(a-c, a-\beta, t)=N(a-c, a-(s a+(1-s) b), t) \\
& =N\left(a-c, a-b, \frac{t}{|1-s|}\right) \\
& =N\left(a-c, b-c, \frac{t}{|1-s|}\right) .
\end{aligned}
$$

Similarly, we have

$$
N(a-c, b-c, t)=N\left(a-c, b-c, \frac{t}{|s|}\right),
$$

that is,

$$
N(a-c, b-c, t)=N\left(a-c, b-c, \frac{t}{|1-s|}\right)=N\left(a-c, b-c, \frac{t}{|s|}\right) .
$$

We note that

$$
\begin{aligned}
& N\left(a-c+a-c, b-c, \max \left\{\frac{t}{|s|}, \frac{t}{|1-s|}\right\}\right) \\
& \quad \geq \min \left\{N\left(a-c, b-c, \frac{t}{|s|}\right), N\left(a-c, b-c, \frac{t}{|1-s|}\right)\right\} \\
& \quad=N\left(a-c, b-c, \frac{t}{|s|}\right)=N\left(a-c, b-c, \frac{t}{|1-s|}\right),
\end{aligned}
$$


and

$$
\begin{gathered}
N\left(a-c+a-c, b-c, \max \left\{\frac{t}{|s|}, \frac{t}{|1-s|}\right\}\right) \\
=N\left(2(a-c), b-c, \max \left\{\frac{t}{|s|}, \frac{t}{|1-s|}\right\}\right) \\
=N\left(a-c, b-c, \max \left\{\frac{t}{|s|}, \frac{t}{|1-s|}\right\}\right) .
\end{gathered}
$$

The previous note implies that

$$
N(a-c, b-c, t)=N\left(a-c, b-c, \frac{t}{|s|}\right)=N\left(a-c, b-c, \frac{t}{|1-s|}\right) .
$$

The strict convexity of $X$ implies that $|s|=|1-s|=1$. Then there exist elements $t_{1}$ and $t_{2}$ in $\mathbb{Z}$ such that $1-s=2^{t_{1}}$ and $s=2^{t_{2}}$. Since $2^{t_{1}}+2^{t_{2}}=1$, we know that $t_{1}, t_{2}<0$. Without loss of generality, we let $1-s=2^{-n_{1}}$ and $s=2^{-n_{2}}$ with $n_{1} \geq n_{2}$. If $n_{1} \gtrless n_{2}$, then

$$
1=2^{-n_{1}}+2^{-n_{2}}=2^{-n_{1}}\left(1+2^{n_{1}-n_{2}}\right) .
$$

Hence $2^{n_{1}}=1+2^{n_{1}-n_{2}}$. This is a contradiction. Thus $n_{1}=n_{2}$, that is, $s=\frac{1}{2}$. This implies that $\beta=\frac{a+b}{2}=\alpha$. Therefore the proof is completed.

Theorem 2.3 Let $X$ and $Y$ be non-Archimedean fuzzy 2-normed spaces over a linear ordered non-Archimedean field $\mathcal{K}$ with $\mathcal{C}=\left\{2^{n} \mid n \in \mathbb{Z}\right\}$. Let $X$ and $Y$ be strict convexities. Suppose that $f: X \longrightarrow Y$ is a fuzzy 2-isometry satisfying that $f(a), f(b)$ and $f(c)$ are collinear when $a, b$ and $c$ are collinear. Then $f(x)-f(0)$ is additive.

Proof Let $g(x)=f(x)-f(0)$. Since $f$ is a fuzzy 2-isometry, so is $g$. It is easy to show that if $a, b$ and $c$ are collinear, then $g(a), g(b)$ and $g(c)$ are collinear. Since $g: X \longrightarrow Y$ is a fuzzy 2-isometry, we have

$$
\begin{aligned}
N\left(g(a)-g(c), g(a)-g\left(\frac{a+b}{2}\right), t\right) & =N\left(a-c, a-\frac{a+b}{2}, t\right) \\
& =N(a-c, a-b, t)=N(a-c, b-c, t) \\
& =N(g(a)-g(c), g(b)-g(c), t) .
\end{aligned}
$$

Similarly, we get $N\left(g(b)-g\left(\frac{a+b}{2}\right), g(b)-g(c), t\right)=N(g(a)-g(c), g(b)-g(c), t)$. Hence

$$
\begin{aligned}
N\left(g(a)-g(c), g(a)-g\left(\frac{a+b}{2}\right), t\right) & =N\left(g(b)-g\left(\frac{a+b}{2}\right), g(b)-g(c), t\right) \\
& =N(g(a)-g(c), g(b)-g(c), t) .
\end{aligned}
$$

By the uniqueness of Lemma 2.2, we have $g\left(\frac{a+b}{2}\right)=\frac{g(a)+g(b)}{2}$ for all $a, b \in X$. Thus $f(x)-f(0)$ is additive, as desired.

Example 2.4 Let $\mathcal{K}=\mathbb{Z}_{3}$, where $\mathbb{Z}_{3}=\{0,1,2\}$. Suppose that the field $\mathcal{K}$ has a nonArchimedean trivial valuation $|\cdot|$. Then $|2|=1$, that is, $\mathcal{C}=\left\{2^{n} \mid n \in \mathbb{Z}\right\}$. 


\section{Acknowledgements}

The authors thank the editor and the referees for their valuable suggestions to improve the quality of this paper. The present research was conducted by the research fund of Dankook university in 2011

Received: 19 November 2012 Accepted: 9 September 2013 Published: 08 Nov 2013

\section{References}

1. Mazur, S, Ulam, S: Sur les transformation isométriques d'espaces vectoriels normés. C. R. Math. Acad. Sci. Paris 194, 946-948 (1932)

2. Baker, JA: Isometries in normed spaces. Am. Math. Mon. 78, 655-658 (1971)

3. Gähler, S: Linear 2-normierte Raume. Math. Nachr. 28, 1-43 (1964)

4. Gähler, S: Untersuchungen über verallgemeinerte $m$-metrische Räume, I, II, III. Math. Nachr. 40, 165-189 (1969)

5. Chu, H-Y: On the Mazur-Ulam problem in linear 2-normed spaces. J. Math. Anal. Appl. 327, 1041-1045 (2007)

6. Moslehian, MS, Sadeghi, G: A Mazur-Ulam theorem in non-Archimedean normed spaces. Nonlinear Anal. 69, 3405-3408 (2008)

7. Mirmostafaee, AK, Moslehian, MS: Stability of additive mappings in non-Archimedean fuzzy normed spaces. Fuzzy Sets Syst. 160, 1643-1652 (2009)

8. Amyari, M, Sadeghi, G: Isometries in non-Archimedean 2-normed spaces. Preprint

9. Katsaras, AK: Fuzzy topological vector spaces II. Fuzzy Sets Syst. 12, 143-154 (1984)

10. Wu, C, Fang, J: Fuzzy generalization of Kolmogoroffs theorem. J. Harbin Inst. Technol. 1, 1-7 (1984)

11. Narayanan, A, Vijayabalaji, S: Fuzzy n-normed linear spaces. Int. J. Math. Math. Sci. 2005(24), 3963-3977 (2005)

12. Narici, L, Beckenstein, E: Strange terrain-non-Archimedean spaces. Am. Math. Mon. 88, 667-676 (1981)

\section{Submit your manuscript to a SpringerOpen ${ }^{\ominus}$ journal and benefit from:}

- Convenient online submission

Rigorous peer review

- Immediate publication on acceptance

Open access: articles freely available online

- High visibility within the field

- Retaining the copyright to your article 